\title{
Ways to Correct Indications for Surgical Treatment of Atherosclerotic Descending Aortic Aneurysms
}

\author{
Vladimir Petrovich Krylov ${ }^{1}$, Nikolay Andreevich Manak ${ }^{2}$, Valentina Nikolaevna Gayduk ${ }^{1}$, \\ Nadezhda Vladimirovna Mankevich ${ }^{3}$, Leonid Ivanovich Reut ${ }^{1}$ \\ ${ }^{1}$ Vascular Surgery Laboratory, Republican Research and Practical Centre 'Cardiology', Minsk, Belarus \\ ${ }^{2}$ Heart Failure Laboratory, Republican Research and Practical Centre 'Cardiology', Minsk, Belarus \\ ${ }^{3}$ Outpatient Department of Republican Research and Practical Centre 'Cardiology', Minsk, Belarus
}

\section{Email address:}

krylov_v_p@mail.ru (V. P. Krylov), kardiovala@tut.by (V. N. Gayduk), mankevich.nv@mail.ru (N. V. Mankevich), reut.49@mail.ru (L.I. Reut)

\section{To cite this article:}

Vladimir Petrovich Krylov, Nikolay Andreevich Manak, Valentina Nikolaevna Gayduk, Nadezhda Vladimirovna Mankevich, Leonid Ivanovich Reut. Ways to Correct Indications for Surgical Treatment of Atherosclerotic Descending Aortic Aneurysms Advances in Surgical Sciences. Vol. 5, No. 3, 2017, pp. 31-35. doi: 10.11648/j.ass.20170503.11

Received: February 13, 2017; Accepted: March 10, 2017; Published: May 3, 2017

\begin{abstract}
Recent studies suggest that the development of atherosclerotic descending aortic aneurysms (DAA) is a complicated process, which occurs due to a polymorphic nature of aortic wall abnormalities. They overlap and contribute to a wide variety of clinical manifestations. The inflammatory process may be the main reason for a rapid growth and rupture of the aneurysm. It also involves a mechanism of complex adhesion comprising of acute-phase proteins to low-density lipoproteins (LDLPs) as atherosclerotic lesions. This process occurs in the presence of connective tissue disorders, aortic wall focal cystic medianecrosis, poor management of arterial hypertension, aging, and reduced compliance. Therefore, the surgical treatment modality to treat DAA becomes less appealing. Additionally, it is not feasible to make indications for surgery based solely on linear aneurysmal diameter. However, medical correction has no contraindications for use.
\end{abstract}

Keywords: Atherosclerotic Descending Aortic Aneurysms, Surgical and Conservative Treatment, Indications for Surgery, Pathogenesis and Mortality Rate

\section{Introduction}

In the present study, due to the similarity of etiopathogenetic processes involved in, the aortic wall contributing to the lesion, we used a 'mixed' term 'descending aortic aneurysms' (DAA) instead of 'descending thoracic aneurysm (DTA) and abdominal aortic aneurysm (AAA).

Up to $25 \%$ of all DAA cases include DTA, whereas other cases involve abdominal aorta [1-2-3]. Knowing about the danger of aneurysm rupture and having no schemes of restricted medical treatment, applied medicine has favored to surgery. Therefore, it is widely accepted that the scheduled repair of DAA should be performed when the aneurysm diameter is greater than $5.5 \mathrm{~cm}$ or at the dilation rate of 1.0 cm per year $[1-2,4]$.

At the same time, a more in-depth examination of the individuals with aneurysm showed the presence of multiple medical conditions, with cardiovascular diseases at the forefront. The abovementioned factors further increase already high mortality after aortic prosthesis operations (valve) by $32-60 \%$ [5]. In this respect, Rachael $O$ et al. [6] believe that the risks associated with the operative treatment and 30-day mortality rate up to $5 \%$ require improvements in individualized diagnostic and therapeutic approaches to treatment in these patients. This is a key task that can promote a better clinical outcome in the presence of risk factors and more significant perioperative changes.

Endovascular treatment methods in DAA subjects are an alternative to a conventional surgical approach. However, EVAR 1 [7] and EVAR-2 [8] trials showed that the number of deaths due to aneurysm ruptures in the group where patients were treated conservatively was consistent with a number of ruptures and post-operation mortality in the group of 
endovascular treatment. There were no differences between the key studied parameters, which authors believe to be a result of a reduced rupture rate in conservative treatment group.

We previously offered the sparing management of these patients implicating conservative treatment and surgery performed only when there is a risk of rupture [9-10-11].

Objectives: to establish factors affecting the mortality rate in individuals with DAA and to develop the means to correct them.

\section{Materials and Methods}

\subsection{Patient Characteristics}

A total of 399 individuals with DAA received treatment in the Republican Research and Practical Centre "Cardiology" for 6 years. They were distributed into three groups. Group 1 (controls) included 63 patients who had received standard treatment in Cardiac Surgery Dpt. 1 in the period between 2010 and 2011 and they were studied retrospectively. Fifty received aortic prostheses, and 6 received a stent-graft. Seven subjects received no surgery due to a high-risk existing comorbidity.

Prospective analysis was made in 121 subjects with a 2year follow-up period (starting from 2012) who entered the Group 2; 99 subjects with a 4-year follow-up (Group 3) and 97 subjects with 5-year follow-up (Group 4). All patients were on file at the consultation departments. They received a standardized, preliminarily modified and verified scheme of DAA management when surgery was embarked on only if there was a peril of rupture [9-10].

\subsection{Methods}

1. Clinical investigations, including CT angiography, aortic angiogrpahy, coronary angiogrpahy, and ultrasonographic methods.

2. Surgical treatment included aortic ileac femoral bifurcation prosthesis and endovascular stent-graft implantation.

3. Medical therapy for aortic strengthening. Permanent ACE inhibitors with amlodipine, b-blockers, statins; and courses of macrolide antibiotics (roxithromycin, azithromycin) when there are signs of inflammatory process (as evidence by CRP), and symptomatic therapy. The CRP was measured from venous blood in $\mathrm{mg} / \mathrm{l}$ by latex-enhanced turbidimetric method using Cobas JNTEGRA 400 analyzer and the testing system produced by Roche Diagnostics (Switzerland).

4. Results were processed using Statistica 5.5 StatSoftCorp. (USA) software for medical-biological research. Estimates of the parameters studied have been expressed as arithmetic mean (M) and average error mean (m). To define statistical significance between the parameters, a Student's $t$ - test was used. Differences were considered significant when the statistical significance $P$ was less than 0.05 .

\section{Results}

The groups of subjects with DAA under trial were not statistically different by age, sex, aneurysm diameter, including those more or less than $50 \mathrm{~mm}$, length, and thoracic and abdominal site. A multifactorial 5-year reduction in the quantity of subjects have not affected statistical data. However, there was a difference between minor, moderate and large sizes of aneurysms. There was a trend to reduced percentage of subjects with aneurysm diameter $<50 \mathrm{~mm}$ in Groups 3 and 4.

Patient management is presented in Table 1.

Table 1. Comparison of treatment modalities in DAA subjects.

\begin{tabular}{llllll}
\hline $\begin{array}{l}\text { Groups, } \\
\text { No. of patients }\end{array}$ & Follow-up & Treatment Modality & $\begin{array}{l}\text { Total no. of operated pts. } \\
\text { including endoprosthesis }\end{array}$ & Nonoperated \\
\cline { 5 - 6 } & & & $88.9 \%(56)-6$ & $11.1 \%(7)$ \\
$2 . n=63$ & 2 yrs. & surgical & $14.0 \%(17)-8$ & $86.0 \%(104)$ & $28.9 \%(35)$ \\
$3 . n=99$ & 2 yrs. & sparing & $24,2 \%(24)-8$ & $75.8 \%(75)$ & $31.3 \%(31)$ \\
$4 . n=97$ & 4 yrs. & sparing & $24.7 \%(24)-8$ & $75.3 \%(73)$ & $33 \%(32)$ \\
\hline
\end{tabular}

Vast majority of (88.9\%) subjects of Group 1 (active surgical approach) were operated. Fifty individuals received aortic replacement surgery and 6 were implanted with the stent-graft. Seven subjects received no surgery due to severe condition secondary to a pronounced comorbidity. In Groups of sparing treatment, surgical intervention was offered in 14\% subjects (Group 2), 24.2\% (Group 3) and 24.7\% (Group 4). Thus, not a single operation has been performed at 5 years now in this cohort, and no cases of aneurysm rupture have been registered.

In Group 2, Group 3 and Group 4, 28.9\% 31.3\% and 33\% subjects were not operated due to severe state, which is more than 3 times more than in Group 1 (11.1\%). More than half of subjects with sparing treatment received no operation as medical treatment ceased the progression of the disease. Although, there is a correlation between aneurysm diameter and threat of rupture, statistical data confirm that the ruptures are possible even when the diameter is less than $40 \mathrm{~mm}$, which we observed three times in our clinical settings. In these circumstances, we registered a pronounced inflammatory process.

All-cause mortality comparison between Groups 1 and 2 has demonstrated that it was 2 times higher when the active surgical approach was used. In Groups of sparing treatment, the similar mortality values become apparent only at 4 years, and improved to $30.9 \%$.

The intergroup comparison of mortality causes showed that more than half of the outcomes were related to a co- 
existing terminal disease, which progresses during DAA development, and with age. Precisely comorbidities are the most life threatening for subjects with DAA unlike aneurysm ruptures which account for only $30.8 \%, 33.3 \%, 34.8 \%$ and $26.7 \%$ in Group 1, 2, 3 and 4, respectively. This value is almost 2 times lower than the values due to concomitant diseases.

Mortality analysis in the Groups under study subject to sparing or surgical treatment modality is presented in Table 2.

Table 2. Mortality analysis in the studied Groups subject to either sparing, or surgical treatment modality.

\begin{tabular}{|c|c|c|c|c|c|c|}
\hline \multirow{2}{*}{$\begin{array}{l}\text { Groups, no. of patients } \\
\text { Follow-up period }\end{array}$} & \multirow{2}{*}{$\begin{array}{l}\text { All-cause } \\
\text { mortality }\end{array}$} & \multirow{2}{*}{$\begin{array}{l}\text { Conservative } \\
\text { treatment }\end{array}$} & \multirow{2}{*}{$\begin{array}{l}\text { Surgical treatment, } \\
\text { including operative } \\
\text { mortality }\end{array}$} & \multicolumn{2}{|l|}{ Due to aneurysm rupture } & \multirow[t]{2}{*}{$\begin{array}{l}\text { Due to other } \\
\text { diseases }\end{array}$} \\
\hline & & & & Conservatively treated & Post-operation & \\
\hline $\begin{array}{l}\text { 1. } n=63 \\
2 \text { yrs. }\end{array}$ & $20.6 \%-13$ & $38.5 \%-5$ & $\begin{array}{l}61,5 \%-8 \\
(15.4 \%-2)\end{array}$ & $15.4 \%-2$ & $15.4 \%-2$ & $53.8 \%-7$ \\
\hline $\begin{array}{l}2 . \mathrm{n}=121 \\
2 \mathrm{yrs} .\end{array}$ & $9.9 \%-12$ & $91.7 \%-11$ & $\begin{array}{l}8.3 \%-1 \\
(8.3 \% 1)\end{array}$ & $33.3 \%-4$ & 0 & $58.3 \%-7$ \\
\hline $\begin{array}{l}3 . n=99 \\
4 \text { yrs. }\end{array}$ & $23.2 \%-23$ & $78.3 \%-18$ & $\begin{array}{l}21.7 \%-5 \\
(8.7 \%-2)\end{array}$ & $26.1 \%-6$ & $8.7 \%-2$ & $56.2 \%-13$ \\
\hline $\begin{array}{l}\text { 4. } \mathrm{n}=97 \\
5 \mathrm{yrs} .\end{array}$ & $30.9 \%-30$ & $80 \%-24$ & $\begin{array}{l}20 \%-6 \\
(8.3 \%-2)\end{array}$ & $8,2 \%-6$ & $8.3 \%-2$ & $66.7 \%-20$ \\
\hline
\end{tabular}

However, the most important thing is that the surgical treatment makes no warranty against aortic rupture. Thus, in the analyzed groups, $8.7 \%$ - $15.4 \%$ subjects had aortic rupture in late postoperative period with two causal factors. The former is a need to provide complex ethiopathogenetic treatment in the postoperative period to address inflammatory process and to strengthen aortic wall. The latter is a lack of method to define integrity threshold limits for aortic wall located proximally or distally to the aneurysms. Visual and palpatory intraoperative assessment of this area will inevitably make surgeon to 'narrow' the area to be operated for the sake of risk reduction of complications.

In conservative treatment, aneurysm ruptures occurred mainly in individuals who were rejected in operation due to a high risk. Most of them showed poor compliance. Comparison of surgical vs. conservative treatment results showed significant differences between Group 1 and the remaining groups. Thus, in active surgical treatment (Group 1 ), the mortality rate was $61.5 \%$ and $38.5 \%$ in subjects treated conservatively. It seems inappropriate to compare this data with that obtained from other groups, as these 63 subjects were referred to an in-patient facility to receive operation, which means that they, including seven more subjects rejected in operation, were somatically healthier than other subjects. Still, five of them died at 2 years due to other diseases.

The highest mortality rates were observed in Group 2, Group 3 and Group 4 among non-operated patients (91.7\%, $78.3 \%$ and $80 \%$, respectively) due to multiple comorbidities, who had been previously rejected in operative treatment.

While analyzing the above-mentioned tables, one has an impression that the mortality rate among non-operated subjects was lower than in those treated conservatively. That's not quite true. It must be concerned that the operation itself somewhat jeopardizes the patient's life. Therefore, one should address the mortality due to rupture together with mortality respective of the treatment method used, i.e. operation. If summarize mortality rates due to rupture with operative mortality, we can have the following: $30.8 \%, 8.3 \%$, $17.4 \%$ and $13.4 \%$ for Group 1, Group 2, Group 3, and Group
4, which is consistent with the mortality estimates observed in conservatively treated individuals $(15.4 \%, 33.3 \%, 26.1 \%$ and $20 \%$, respectively).

To define pathogenetic factors to cause DAA, we excluded the subjects from Group 3 (4-year follow-up) who missed visits for control measurements. The remaining patients were distributed into two subgroups. Subgroup 1 included those who had not shown $5 \mathrm{~mm}$ aneurysm diameter enlargement in 6 months. Subgroup 2 included patients with apparent progression of the disease [10].

Data analysis including 69 studied parameters suggested that these subgroups had statistical significance for a variety of these parameters. Subgroup 1 showed CRP $<2.7 \mathrm{mg} / \mathrm{l}$; LDLPs $<2.7 \mathrm{mmol} / \mathrm{l}$; atherogenic index $<2.68$, aneurysm diameter (d) $<36.3 \mathrm{~mm}$ and $\mathrm{BP}<132.4178 .6 \mathrm{~mm} \mathrm{Hg}$. Subjects with these indices were referred to as having favorable course of the disease. Negative dynamics was identified in those with $\mathrm{CRP}>8.3 \mathrm{mg} / \mathrm{l}$ at baseline; aneurysm diameter $>44.3 \mathrm{~mm}$; LDLPs $>3.46 \mathrm{mmol} / \mathrm{l}$; atherogenic index $>4.05 ; \quad \mathrm{BP}>142.1 / 86.77 \mathrm{~mm} \mathrm{Hg}$., and with the decreased compliance. These subjects were referred to as having adverse course, and a differentiated medical treatment with mandatory wall-strengthening drug administration.

\section{Discussion}

Our investigations including a small number of subjects made it difficult to review descending thoracic and abdominal aortic aneurysms separately. Based on the similarity of atherosclerotic and inflammatory lesions, we considered them collectively, and we observed no specificities related to manifestation irrespective of the site. Atherosclerotic aneurysms of descending thoracic segment were registered in Groups of sparing treatment in 6 and 8 subjects, whereas 14 other subjects had thoracoabdominal aneurysms.

Many sources [12-13-14] consider, that thoracic aneurysm are more severe. Based on this data, 5- year mortality in subjects with DTA is lower compared to AAA (64\% and $75 \%$, respectively). In our Groups, we had no such 
observations. In our opinion, it can be explained by the fact that targeted medical treatments block the factors contributing to aneurysmal aortic wall destruction. The underlying cause of mortality is therefore a comorbidity, which becomes further activated in patients with juxtarenal and juxtavesical segment involvement, which is frequently the case in AAA. Thus, two-thirds of these subjects show silent chronic kidney disease (CKD) or abdominal events even when the aneurysm location is $2 \mathrm{~cm}$ or more from renal arteries.

The course of AAA is somewhat exacerbated by structural heterogeneity of descending aortic segments. Thoracic aortic intima media thickness reaches 55-60 laminar units and this segment of aorta has both avascular and vascularizing zones, while AAA media is totally avascular as it consists of 28-32 laminar units [15]. In DTA, media thickness increases at birth of a patient due to the synthesis of laminar units, whereas in AAA due to the enlargement of the existing units. Therefore, the aortic segment having fewer laminar units and worse supply conditions is more subjects to media degradation and aneurysm formation.

Recent studies suggest that the concept about primary nature of atherosclerosis in DAA development is quite doubtful. It may be present as a syndrome that is detrimental to aneurysm morphology or it may tighten the aortic wall.

Rachel et al. [6] differentiate the signs of abdominal aneurysm and atherosclerosis formation. They include intraluminal partial thrombosis, proteolytic elastin and collagen degradation, involvement of mainly tunica externa contributing to vascular dilation, necrotic inflammation, lymphocyte and macrophage infiltration, and mural calcification. As for the manifestation of atherosclerosis, it includes deposit of fat and intimal plaque formation, thrombosis at the site of rupture in plaque area, positive luminal remodeling, intimal calcification and dilation resulting in intraluminal stenosis.

Comparison of those abnormalities suggests that despite the presence of similar pathologic processes, they are different. For example, atherosclerosis mainly affects intimal vessels, while aneurysmal dilation is prone to media and adventitia destruction. As a result, clinical evidence of the disease (clinical manifestation) implicates the aortic wall dilation with rupture potential with simultaneous atherosclerotic process leading to stenosis.

Based on data presented by Luneva et al. [12], intimal thickening was characteristic of atherosclerotic lesion due to extracellular lipid accumulation lakes with massive lymphocyte, macrophage and foam cell infiltration, which is clearly consistent with the inflammatory process, infection sings, and thrombosis or the absence of thrombosis.

Clinical manifestations of the disease seem to be of mosaic nature with any of lesion factor being the major factor of tissue destruction, or in contrast, making a compensatory (protective) effect in response to a potential fatal outcome. Something similar was observe in 8 of our subjects whose aneurysms were $>50 \mathrm{~mm}$ and aortic lumen diameter $8-12 \mathrm{~mm}$ suggesting the presence of concomitant processes of dilation and occlusion involving all aortic strata.

Some literature sources [12] suggest that in $15 \%$ of operated patients no histological abnormalities were identified in operation zones suggesting the structurally impaired vascular wall currently unavailable for analysis. Authors, therefore, consider that the clinical diagnosis is not always consistent with the results obtained from morphological analysis necessitating development and usage of all options available for diagnosis.

They argue that cystic medianecrosis is more frequently (62\% of cases) identified in the descending aortic segments. The development of cystic medianecrosis is believed to be related with both connective tissue disorders, and its degradation with age dictated by arterial hypertension $(\mathrm{AH})$ development [16]. These changes were localized in media as impaired tubular architecture caused by ruptures, elastic fiber lysis and cysts secondary to necrotic foci and cell-free fibrosis. However, the authors believe that the cystic medianecrosis is probably the sign of the frailty of the connective tissue.

\section{Conclusion}

Differential diagnosis and treatment of DAA currently represents challenge due to polymorphous nature of processes occurring in the aortic wall, which overlapping make a variety of clinical manifestations, and each of them need, to considered and addressed. Therefore, it is time to shift from a too simplified assessment of DAA progression, such as the aneurysm diameter offering no integral view of pathological changes involved.

The growth of DAA is not linear. The rupture may appear in either minor diameters or large aneurysms [17,6]. Thus, the search of biomarkers that might be indicative of the risk of rupture irrespective of aneurysm diameter is crucial.

It is becoming clear that the key pathobiological processes of DAA progression and the ruptures include neovascularization, necrotic inflammation, calcification, extracellular matrix proteolytic degradation, connective tissue dysfunction, and other abnormalities. All of them may play a crucial role in the development of methods of direct and indirect imaging that could be helpful in the assessment of aneurysm enlargement or rupture.

Rachael et al [6] have shown that iron oxide subminiature superparamagnetic particles are able to grasp and identify regions of macrophage activity. Thus, positron-emission tomography identifies regions of inflammation, angiogenesis, hypoxia and macrocalcification, allowing for a better prognosis of aneurysm enlargement and rupture. Ability to control the increased activity of macrophages, collagen and elastin proteolytic degradation allows for identification of key components of AHA formation, as well as for a development of optimal approaches to medical correction.

Authors have shown that some regions of the aneurysmatic aortic wall may show focal and intensive biological activity with proteolysis and macrophage infiltration. In this case, the aortic wall distensibility threshold becomes uneven and is impaired 
predominantly in the areas of increased proteolytic activity, which varies due to the presence of aneurismal sac. These biological 'hotspots' obviously represent the regions of the aortic walls prone to further dilation and rupture considering specific features of zonal hemodynamics [18-19-20].

Plenty macrophages present in the aneurysmatic aortic wall suggest that this might be a reliable indicator of some processes involved in this area [6]. Our studies allowed making such conclusion based on the identification of parameters for a favorable and unfavorable course with CRP being the key criteria. With CRP estimates more than $2.7 \mathrm{mg} / 1$, we administered azithromycin or doxycycline resulting in a quick management of the inflammation in most cases. While using complex treatment based on the existing dyslipidemia, $\mathrm{AH}$, comorbidities, advanced age and surgical activity in $20 \%$ in the group with a 5 -year follow-up period, we observed aneurysm ruptures in $8.2 \%$ subjects with similar proportions in both operated and non-operated patients.

Thus, the analysis of our studies and published investigations suggests that the formation of the descending aortic atherosclerotic aneurysms represents a multifactorial process. Each factor can appear depending on the connective tissue conditions, trigger factors including inflammatory, infectious, traumatic, and chemical and others; severity of $\mathrm{AH}$, dyslipidemia and compliance, as well as combinations of those.

In this relation, it seems unfeasible to take the linear indicator, which is aneurysm diameter as a criterion for operative treatment as it does not address the multifactorial nature of DAA development and progression. The clinical variety of the disease, a large number of comorbidities, new options of medical treatment with the management of key factors involved in the lesions afford to broadly implement conservative approaches to DAA treatment.

\section{References}

[1] Shirinbek O. Infrarenal thoracic aneurysms: current modalities and treatment outcomes (Literature Review) // The Bulletin of A. N. Bakoulev Scientific Center for Cardiovascular Surgery of Russian Academy of Medical Sciences CARDIOVASCULAR DISEASES. - 2008 - Vol. 9, No. 5.pp. 50-57.

[2] Hallett J. W. Jr. Abdominal aortic aneurysm: natural history and treatment // Heart Dis. Stroke. - 1992. -Vol. 1. - P. 303308 .

[3] Lower Extremities Artery and Abdominal Aortic Aneurysm Adult Patient Management National Guidelines (Russian Consensus Paper), M: Bakoulev Scientific Center for Cardiovascular Surgery of Russian Academy of Medical Sciences, pp. 2011. - 140 .

[4] Bokeria L. A. (2010) Thoracic and Thoracoabdominal Aortic Aneurysm Surgery: Guidelines for Healthcare Professionals / L. A. Bokeria, V. S. Arakelyan - M.: Bakoulev Scientific Center for Cardiovascular Surgery of Russian Academy of Medical Sciences. 394.

[5] Boersma E. (2005) Perioperative cardiovascular mortality in non-cardiac surgery: validation of the Lee cardiac risk index/E. Boersma, M. D. Kertai, O. Schouten et al. //Amer. J. Med. 118, 1134-1141.

[6] Rachael O Forsythe, David E Newby, Jennifer M J Robson. (2016), Monitoring the biological activity of abdominalaortic aneurysms Beyond Ultrasound. 6, 817-824.

[7] EVAR trial participants. (2005) Endovascular aneurysm repair versus open repair in patients with abdominal aortic aneurysm (EVAR trial 1): randomized controlled trial $\mid$ Ibid/-. 365. 2179-2186.

[8] EVAR trial participants. (2005) Endovascular aneurysm repair and outcome in patients unfit for open repair of abdominal aortic aneurysm (EVAR trial 2): randomized controlled trial $\backslash$ Ibid. 365. 2187-2192.

[9] Krylov V. P., Mrochek A. G., Titov L. P., Gaiduk V. N., Reut L. I., Smaliakou A. L. Can We Change a Look at Atherosclerotic Aortic Aneurism Treatment? Health, 2014, p 1345-1351.

[10] Mankevich N. V., Mrochek A. G., Krylov V. P., Gayduk V. N., Reut L. I., Smoliakou A. L. Predictors of atherosclerotic descending aortic aneurysms and correction. 'Kardiologiia $\mathrm{v}$ Belarusi', No. 5 (42), 2015 - pp.63-73.

[11] Krylov V. P., Titov L. P., Gayduk V. N., Reut L. I., Smaliakou A. L., Mankevich N. V. Atherosclerotic descending aortic aneurysm. Pros and cons of surgery\ World Journal of Cardiovascular surgery, 2015, 5, 91-101.

[12] Luneva E. B., Uspensky V. E., Mitrophanov L. B. et al. Factors contributing to thoracic aortic aneurysm development. 'Rossiiski kardiologichesky zhurnal' 2013, 1 (99), pp. 19-22.

[13] Cozjnsen L., Braam R. L., Waalewijn R/ et al. What is new in dilatation of the ascending aorta? Review of current literature and practical advice for the cardiologist. Circulation, 2011; 123: 924-8.

[14] Lederle F. A., Wilson S. E., Johnson G. R. et al. Aneurysm detection and management Veterans Affairs cooperative study group. Immediate repair compared with surveillance of small abdominal aortic aneurysm $\backslash$ N. Engl. J. Med. 2002, 346. $1437-1444$

[15] Yuan S., Jing H. Cystic medial necrosis: pathological findings and clinical implications. Rev Bras Cir Cardiovasc. 2011; 26. $1: 107-15$.

[16] Libbi Peter, Bonow P., Mann D. et al. Braunwald's Heart Disease: A Textbook of Cardiovascular Medicine. Elsevier. 2010, Chapter 56:1464.

[17] Nicholls S. C, Gardner J. B, Meissner M. H, et al. Rupture in small abdominal aortic aneurysms. J Vasc Surg 1998; 28: 884-8.

[18] Naghavi M, Libby P, Falk E, et al. From vulnerable plaque to vulnerable patient's call for new definitions and risk assessment strategies: Part II. Circulation 2003; 108: 1772-8.

[19] Thompson MM, Jones L, Nasim A, et al. Angiogenesis in abdominal aortic aneurysms. Eur J Vasc Endovasc Surg 1996; 11: 464-9.

[20] Vallabhaneni SR, Gilling-Smith GL, How TV, et al. Heterogeneity of tensile strength and matrix metalloproteinase activity in the wall of abdominal aortic aneurysms. J Endovasc Ther 2004; 11:494-502. 